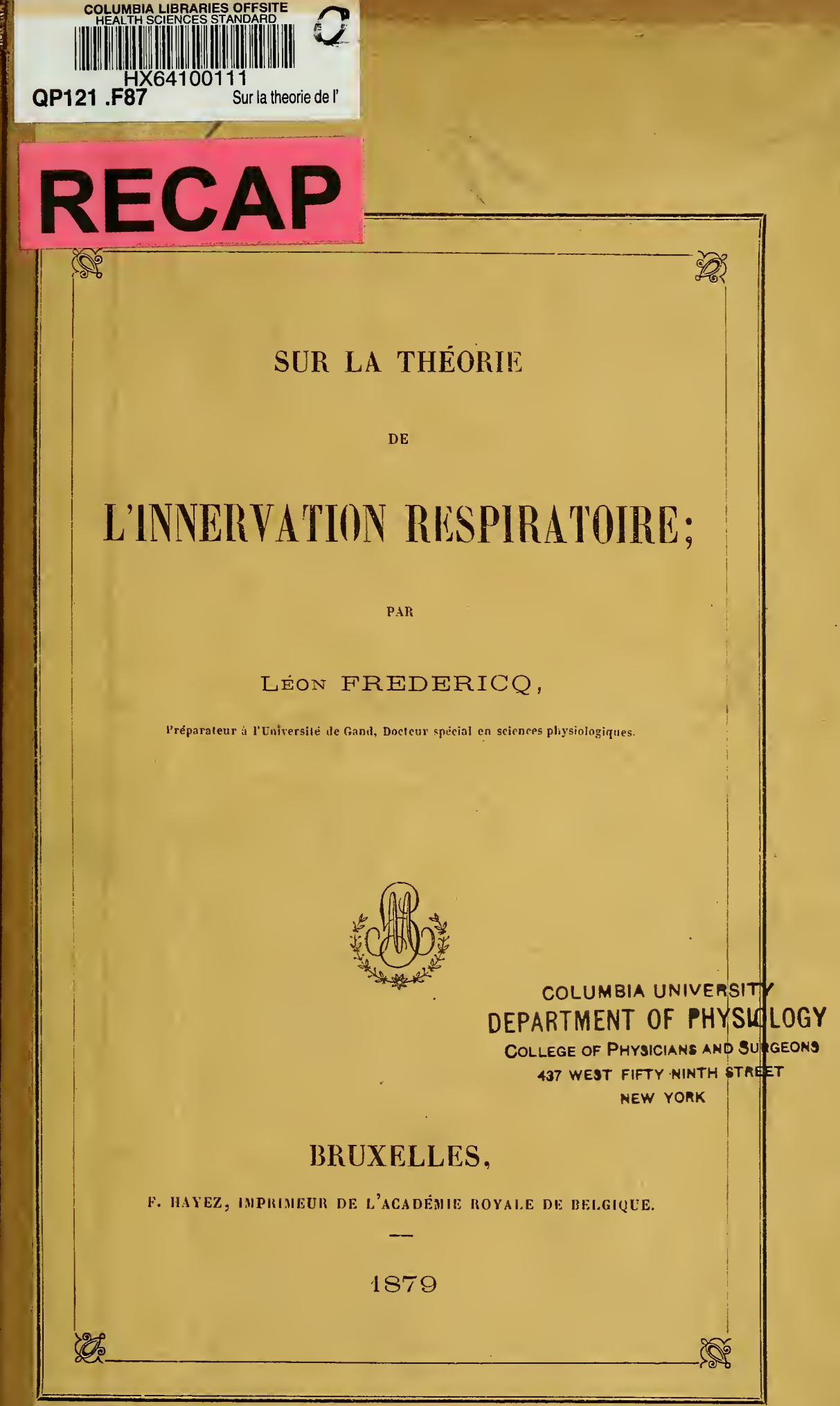




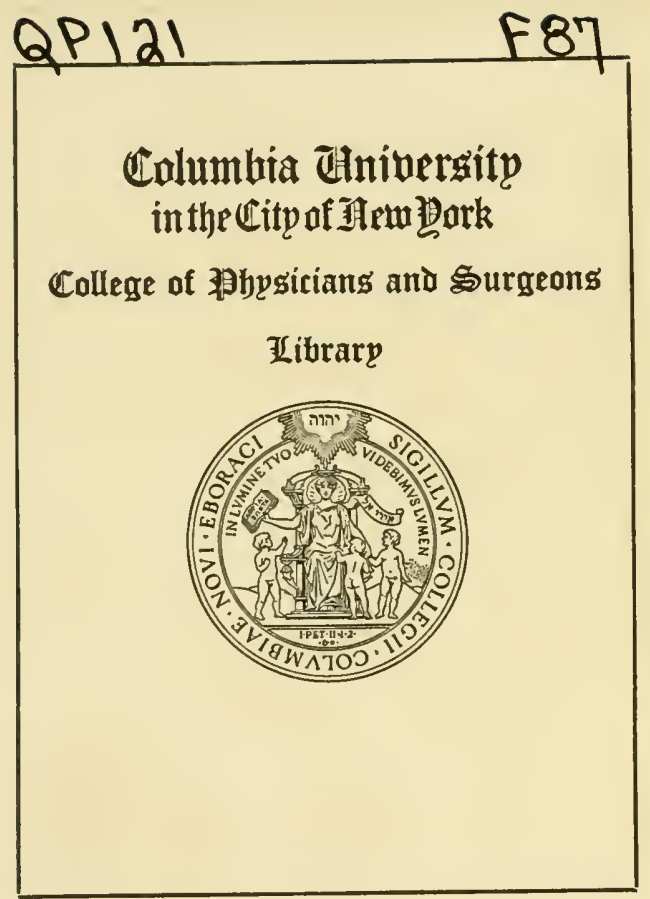


Digitized by the Internet Archive in 2010 with funding from

Open Knowledge Commons (for the Medical Heritage Library project) 



\section{SUR LA THÉORIE}

DE

\section{L'INNERVATION RESPIRATOIRE;}

PAR

LÉON FREDERICQ,

Préparateur à l'Université de Gand, Docteur spécial en sciences plyysiologiques.

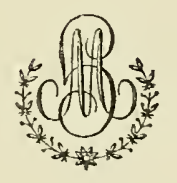

\section{BRUXELLES,}

F. HAYEZ, IMPRIMEUR DE L'ACADÉMIE ROYALE DE BELGIQUE.

1879 


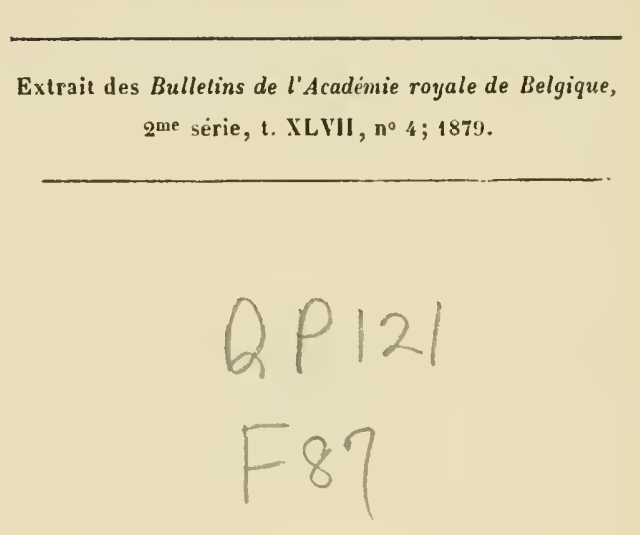




\section{SUR LA THĖORIE}

DE

\section{L'INNERVATION RESPIRATOIRE.}

Les mouvements des muscles respiratoires de la face, du larynx et du tronc ont leur centre dans une portion limitée de la moelle allongée que Flourens a nommée nœud vital : la destruction de ce centre arrête immédiatement la respiration. D'autre part, on peut isoler la région du nœud vital du reste du système nerveux par la section de la moelle pratiquée immédiatement en dessous, sans supprimer les mouvements respiratoires de la face (ouverture des narines = inspiration; abaissement des narines = expiration). On peut faire l'opération inverse, isoler le nœud vital des centres situés au-dessus de lui, en pratiquant l'ablation du cerveau et du cervelet; les mouvements respiratoires n'en continuent pas moins, ceux de la face exceptés. Il semble donc superflu pour expliquer leur production rhythmée, d'invoquer l'action d'impressions sensitives venues du dehors. Ce sont des mouvements automaTIQUES et non des mouvements RéFLEXES. 


\section{(4)}

Rosenthal (1) a montré que le stimulus sous l'influence duquel le centre respiratoire exerce son activité, doit être cherché dans un certain degré de vénosilé du sang qui le baigne. Il s'agirait à la fois d'un déficit d'oxygène et d'un excès de $\mathrm{CO}_{2}$ d'après les travaux de Dolımen et de Pnẗ̈ger.

Si le sang est saturé d'oxygène, s'il est en mème temps parre en $\mathrm{CO}_{2}$, il n'agit plus comme excitant sur le centre respiratoire dont l'activité s'arrête momentanément, l'animal cesse de respirer jusqu’à ce que son sang ait de nouveau acquis le degré de vénosité qui constitue le stimulus (Apnée). S'il est trop veineux par artérialisation du sang, le centre respiratoire se trouve trop virement excité et l’animal exécute des mourements respiratoires exagérés (Dyspnée, gène respiratoire).

Tous ees faits ont été vérifiés un grand nombre de fois et sont devenus pour ainsi dire classiques (2).

(1) Rosenthal. Die Athembewegungen. Berlin 1862, p. 256.

"Die Athembewegungen verden erregt durch den Reiz des Blutes " auf das respiratorische Centralorgan. Der Uebergang dieser Erre"gung auf die betreffenden Nerven und Muskeln findet einen Wider"sland, durch welchen die stetige Erregung in eine rhylhmische Action " umgeselst wird. Dieser. Viderstand wird vermindert dureh die Ein" wirkung des N. vagus, vermehrl dureh die Eimwirkung des N.laryn" geus superior. Der Grad der Thaitigkeit des Centralorgans ist abhän" gig von dem Sauerstoffgehall des Blules, die V'ertheilung diestr

"Thätigkeit auf einzelne Respirationen (und demgemäss die Zahl und

"Tiefe derselben bei gleichbleibender Erregung) von der Wirkung

" jener Nerven."

Stutien über die Athembewegungen. Archis für Anatomie, 186.1, p. $40 \% 6$; ibid., 18630 , p. 191; ibid., 1870, p. 425.

(2) Ces lignes étaient écrites quand parurent dans le Zeitschrift firr physiologische Chemie III. 1, les criliques que Iloppe-Seyler adresse à la thèorie de PQüger. 


\section{( 丂 )}

L'excitation que le nœud vital éprouve de la part du sang est une excitation continue, au moins dans les circonstances ordinaires : comment expliquer l'activité intermittente, rhythmée de ce centre. Rosenthal admet que ce centre est gêné dans son activité, qu'il a à vaincre une résistance qui transforme l'excitation continue du sang en une série de décharges, dont chacune provoque un mouvement respiratoire.

On voit de suite que ce centre respiratoire est surlout un centre d'inspiration : l'inspiration dans les conditions ordinaires est en effet la senle phase active, musculaire de la respiration; l'expiration normale n'est que le retrait, l'affaissement passif du thorax et du poumon survenant pendant le repos, la pause qui sépare deux inspirations. L'expiration n'est donc que la suspension du mourcment d'inspiration: un animal mort, de même qu'un animal rendu apnoïque est à l'état d'expiration. On verra plus loin que cette partie de la théorie de Rosenthal s'accorde fort bien avec les faits nouveaux contenus dans cette note; surtout si l'on admet que l'obstacle qu'épronve le centre Inspiratoire pour entrer en activité, provient d'un second centre qui joue vis-à-vis du premier un rôle de modérateur, de centre d'arrêt.

Il est positif que le rhythme intermittent de la respiration ne dépend pas de changements survenant périodiquement dans la composition du sang par le fait même de chaque mouvement respiratoire, comme Rosenbach (1) l'admet pour les cas où les pneumogastriques ont été coupés.

(1) Rosenbach. Studien über den Nerrus vagus. Berlin, 1877. 
En effel les mourements respiratoires rhythmés, ceux de la face continuent encore alors que la circulation est arrétée sur une tète de lapin qu'on vient d'isoler complétement du reste du corps par la section du cou (la moelle doit nécessairement avoir été coupéc au-dessous du nœud vital).

Les centres respiratoires trouvent donc en eux-mèmes et dans la composition du sang tous les éléments nécessaires à leur activité : ils n'en sont pas moins, dans une certaine mesure, sous l'influence du système nerveux périphérique. Ainsi la section de la moelle, celle du nerf phrénique, etc., font baisser le nombre des mourements respiratoires. Hais sous ce rapport aucun nerf n'exerce d'action aussi marquée que le pneumogastrique. Cette action a été étudiée par un très-grand nombre d'expérimentateurs qui malheureusement sont souvent arrivés à des résultats diamétralement opposés.

Mon intention quand j’ai entamé ce sujet n’était nullement de faire un travail spécial sur l'innervation de la respiration, mais seulement de me former une opinion sur quelques-uns des points controversés les plus importants, notamment sur l'existence tant discutée de fibres centripètes expiratoires dans le tronc du pneumogastrique pulmonaire. J'ai répété avec des résultats affirmatifs les expériences que l'on a fait valoir en faveur de leur existence, et comme j'en ai donné de nouvelles preuves, je ne crois pas faire chose inntile en publiant les conclusions ausquelles je suis arrivé.

Toutes mes expériences ont été faites avec l'aide d'appareils enregistreurs : grâce à l'emploi de la méthode graphique, elles se prêtent fort bien à la démonstration devant un nombreux auditoire; ce sont des expériences de cours. 
J'ai successivement employé le kymographe de Ludwig (nouveau modèle) et le cylindre enregistreur de Narey. Dans les deux cas, les mouvements de la respiration étaient transmis à un tambour enregistreur de Marey. Lorsque j'employais le kymographe, le levier inscripteur du tambour était remplacé par une petite tige de hois portant à son extrémité une pipette de verre chargée d'encre, écrivant en noir sur le papier blanc du kymographe. Sous le tracé de la respiration s’inscrit celui du temps; c'est un trait horizontal se relevant pour former un crochet à chaque seconde. Ce mode d'enregistrement offre un grave défaut : la courbe obtenue se trouve déformée sous l'influence de deux causes : le frottement assez fort entre le papier et la plume, puis l'inertie du levier inscripteur qui est eu proportion de sa masse. La commodité que l'on éprouve à écrire sans interruption sur un papier sans fin ne compense pas ces désavantages. Aussi j’ai renoncé bien vite à ce mode d'enregistrement pour recourir uniquement à l'emploi du cylindre de Marey. Le graphique s'obtient ici, comme on sait, par un léger style terminé en pointe effilée qui gratte le noir d'un papier enfumé et y laisse un tracé blanc.

Le papier que j'emploie est glacé à l'acétate de plomb (papier porcelaine), il est tout à fait lisse, il se noircit admirablement sans jamais brûler. Je ne lui connais qu'un seul défaut, c'est de coûter fort cher. Ce papier est collé sur le cylindre et noirci à l'aide d'un rat de la façon ordinaire (voir Marey, La méthode graphique dans les sciences expérimentales, p. 460, Paris 1878). Il n'est pas nécessaire de marquer le temps, le cylindre offrant un monvement très-uniforme, faisant un tour (longueur 42 centimètres) 
en 62 secondes. Chaque centimètre de papier représente done environ $1 \frac{1}{2}$ seconde de durée.

Les expéricnees dont les détails suivent, ont été exécutées au Laboratoire de physiologie de l'Université de Gand. J'ai pu en répétcr quelques-unes au Laboratoire de M. le professcur Marey (Collége de France à Paris) : je le prie de recevoir ici mes remerciments pour la bienveillance qu’il m’a témoignée. MI. le docteur François Franck, sousdirecteur de ce laboratoire, n'a cessé de m'y guider de ses conseils. Je lui dois plusieurs améliorations dans le plan de mes expériences, notamment l'idée d'enregistrer les mouvements du soufflet pour la respiration artificiellc. Je tiens à lui en exprimer toute ma gratitude. J'ai pu également utiliser sous sa direction un soufllet mû par un moteur à cau et offrant par conséquent un mouvement tout à fait uniforme.

La première expérience qui se rapporte au sujet que je traite est déjà ancienne. Traube (1) a découvert que si l'on pratique chez un animal (un lapin, par exemple), la respiration artificielle à l'aide d'un soufflet, le rhythme primitif des mouvements respiratoires (observé aux narines) se modifie de telle sorte qu'il s'accommode complétement au rhythme des insufflations. Le lapin en expérience répond à chaque insufflation par une expiration, et fait une inspiration à chaque intervalle entre deux insufflations; il fait

(1) Traube. Gesammelte Beilräge zur Physiologie u. Pathologie. Bd. I, p. 17j. Je ne connais le Irarail de Traube que par les cilations de Breuer el de Rosenbach. 
donc exactement le même nombre de mouvements que le soufflet et ces mouvements luttent avec ceux du soufflet. On peut accélérer ou ralentir le rhythme de ses respirations, rien qu'en augmentant ou en diminuant le nombre des insufflations. Traube a montré que c'est dans le pneumogastrique que se trouve la voie nerveuse par laquelle l'état de distension du poumon ou du thorax retentit ainsi sur le centre des mouvements respiratoires. Si l'on supprime cette voie par la double section des pneumogastriques, on n'observe plus aucun rapport entre les mouvements respiratoires de l'animal et ceux du soufllet. L'animal continue à respirer sans aucun souci des insulflations. Breuer et Rosenbach ont contirmé ces faits.

J'ai répété l'expérience de Traube sur un chat, sur un chien morphiné, sur un cobaye, sur un jeune lapin qui a vait subi l'ablation des hémisphères cérébraux et sur plusieurs lapins, les uns non anesthésiés, les autres sous l'influence respective du chloral, de la morphine ou du laudanum. Le lapin, surtout s'il est anesthésié par le chloral ( 1 à 5 grammes en injection sous-cutanée), se prête beaucoup mieux à cette expérience que le chien, le chat ou le cobaye. J'avais essayé d'inscrire les mouvements des naseaux de l'animal à l'aide d'un long style de verre fixé par une goutte de cire à cacheter aux poils du lobe médian du nez, mais les résultats obtenus de cette façon ne me satisfirent guère. J'y renonçai bientôt pour adopter la disposition expérimentale suivante :

Le lapin immobilisé sur le support de Czermak est trachéotomisé : on fixe dans la trachée un tube de verre en T. L'une des branches du T est reliée par un tube de caoutchouc à un tambour à levier de Marey, qui inscrit les varia- 
tions de la pression latérale dans la canule trachéale; l'autre branche est mise en rapport avec le tube de caoutchouc qui vient du soufflet et par lequel on pratique les insufflations. Ce tule de caoutchouc porte latéralement tout près de la canule trachéale un petit orifice destiné à laisser échapper l'excédant de l'air et à permettre l'expira. tion à l'animal. Les insufflations se pratiquent à l'aide d'un soufflet à ressort mù par le pied de l'expérinentateur. Une petite poire en caoutchouc logée entre les tours du ressort transmet par l'intermédiaire d'un tube de caoul. chouc les monvements du soufflet à un second tambour à levier dont le style inscripteur trace sa courbe à côté de celle des mouvements de l'air dans la trachée.

Si l'on pratique une série d'insufflations sur un animal dont les pneumogastriques sont intacts, les deux graphiques se correspondent exactement. Or, le tracé fourni par le tambour qui est en rapport avec la canule trachéale de l'animal est évidemment le produit de deux facteurs, savoir : $1^{\circ}$ le courant d'air émanant du soufflet et $2^{\circ}$ celui qui est dû aux mouvements respiratoires de l'animal. Puisque les deux tracés sont semblables, il faut en conclure que les mouvements respiratoires de l'animal suivent exactement les insufflations du soufflet. On doit naturellement s'assurer que l'animal ne cesse pas de respirer, que la ventilation pulmonaire n'est pas suffisante pour amener l'apnée par suroxygénation, artérialisation du sang.

L'inspection des narines de l'animal montre qu'il en est réellement ainsi : les narines s'affaissent à cluaque insufflation (position d'expiration), elles s'ouvrent (position d'inspiration) dans l'intervalle entre deux insufllations. Les 


\section{(11)}

mouvements de l'animal coïncident avec ceus du soufflet, mais se font en sens inverse.

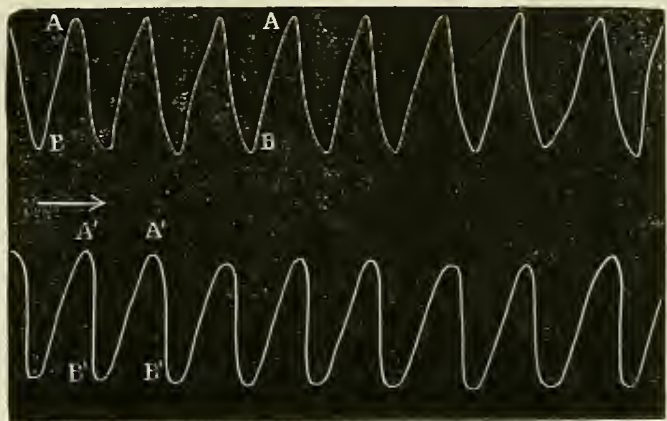

Fig. 1. Tracés simultanés de la pression laterale dans la trachèe (AB) el des excursions du sauffet (A'B'). Lapin.

La correspondance entre les deux graphiques se voit très-bien sur la fig. 1. Le tracé va de gauche à droite suivant la direction de la flèche; il se lit comme une courbe de manomètre inscripteur, c'est-à-dire que les collines $\mathbf{A}, \mathbf{A}^{\prime}$ correspondent aux augmentations de pression (expirations), les vallées $B, B^{\prime}$ aux diminutions de pression (inspirations).

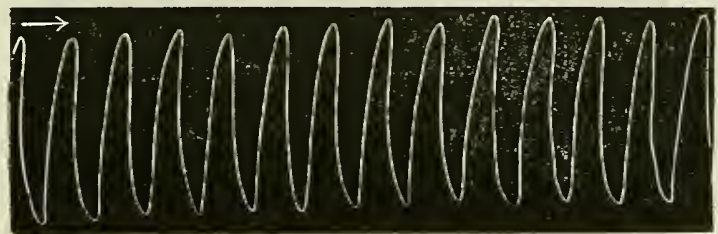

Fig. 2. Graphique de la pression laterale dans la trachee pendant les insufflations du soufflet automatique. Lapin.

Si j'emploie le soufflet actionné par le moteur à eau, ses excursions sont exactement égales entre elles et il n'est plus nécessaire de les inscrire. Le tracé de la pression 
dans la trachée est alors tout à fait régulier. La fig. 2 cn montre un exemple.

La voie par laquelle les excursions du poumon retentissent ainsi sur les centres respiratoires doit être localisée dans le tronc des pneumogastriques. Dès qu'ils sont coupés, l'accord que l'on observait entre les mouvements respiratoires et les insufllations est rompu. Les respirations de l'animal interfèrent avec les mouvements du soufllet. La courbe de la pression de l'air dans la trachée trahit le désaccord entre les deux facteurs qui concourent à la former: les insufflations el les mourements de l'animal.

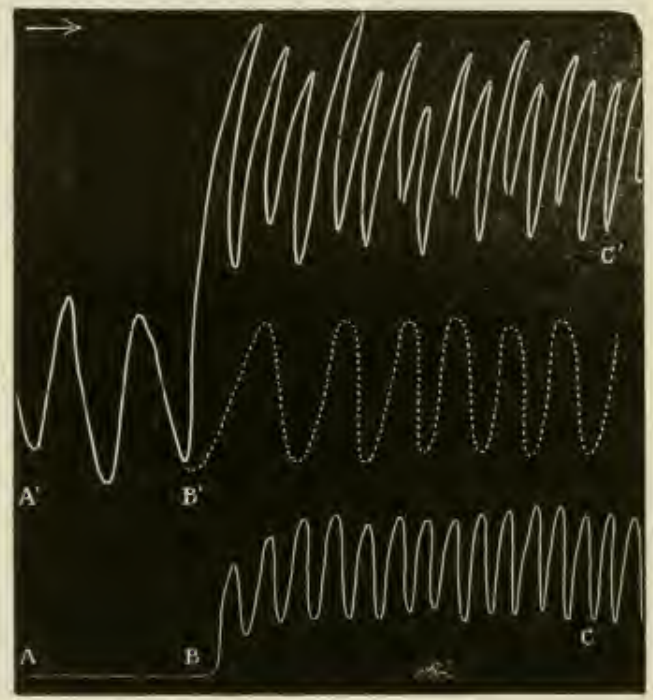

Fig. 5. Lapin à pneumogastriques coupés. ABC. Tracé dic souffel.

$\mathbf{A}^{\prime} \mathbf{B}^{\prime} \mathbf{C}^{\prime}$. Trace de la pression dans la trachee.

La courbe pointillè a été ajoutée à la main : elle est destinée a représenter les mouvements respiraloires de l'animal tels qu'on les aurail oblenus sans faire d'insuffations.

La fig. 3 en donne un exemple. Le graphique inférieur 


\section{(13)}

nous montre que le soufflet est resté au repos de A en B, qu'à partir de $\mathbf{B}$ l'on a pratiqué une série d'insufflations. La courbe supérieure qui représente la pression de l'air dans la trachée indique de $\mathbf{A}^{\prime}$ en $\mathbf{B}^{\prime}$ ce qu'était la respiration de l'animal, pendant le repos du soufflet. Dans la portion $\mathrm{B}^{\prime} \mathrm{C}^{\prime}$ on distingue fort bien à travers les variations dues au mouvement du soufflet, celles provenant des mouvements respiratoires de l'animal. La courbe pointillée a été ajoutée à la main, elle représente les mouvements respiratoires tels qu'on les aurait obtenus sans insufflations.

Le graphique suivant ( $\mathrm{n}^{\circ} 4$ ) est empruuté à la même expérience qui a déjà fourni le tracé $n^{\circ} 2$. Il représente également la pression latérale dans la trachée pendant les insufflations du soufflet aulomatique. La seule condition nouvelle, c'est que les deux pneumogastriques ont été coupés. Dès lors la courbe obtenue n'est plus simple, régulière, quoique les insufflations soient rigoureusement semblables. La courbe pointillée a la même signification que dans la figure 3.

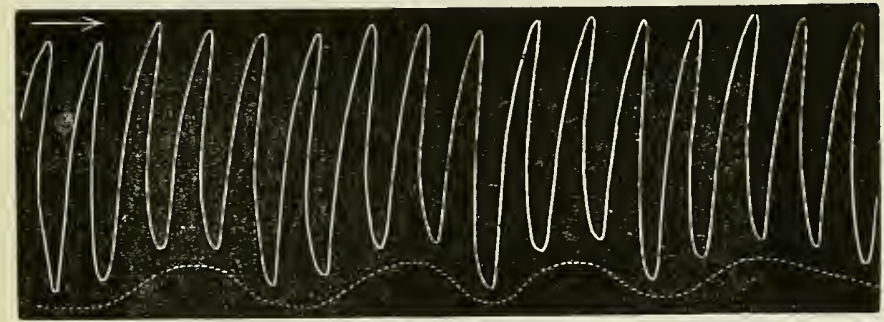

Fig. 4. Graphique de la pression latérale dans la trachèe pendant les insuffations du souffet aulomalique. Lapin à pneumogastriques coupćs La ligne pointillèe à la méme signification que dans la figure $\mathbf{3}$.

Pour obtenir ce résultat, il faut couper les deux pneumogastriques, la section d'un seul ne sulfit pas; aiusi la 
fig. 2 a ėté empruntée à un tracé fourni par un lapin dont un pneumogastrique avait été coupé.

Les expériences de Breuer (1) nous donnent la clef de l'expérience de Traube. Breuer a montré que l'état de distension mécanique du poumon était ici le principal facteur. Chaque insufflation, chaque expansion mécanique du poumon a pour effet de provoquer chez l'animal l'état d'expiration, chaque mouvement de retrait du poumon provoque, au contraire, l'inspiration. C'es lle long des fibres du pneumogastrique que cheminent les excitations centripètes qui provoquent tantôt l'inspiration, tantôt l'expiration. Malgré leur importance capitale, les expériences de Breuer n'ont été répétées qu'un petit nombre de fois (2). L'un des expérimentateurs, Guttmann, est arrivé à des résultats un peu différents. On peut dire que ces expériences de Brener n’ont pas encore passé dans le domaine classique de la physiologie. On me permettra donc de revenir sur celles d'entre elles qui ont donné lieu à des discussions, ce sont les expériences tendant à prouver qu'il existe dans le pneumogastrique des fibres centripètes qui ont pour effet d'arrêter la respiration à l'état d'expiration (active) et qui sont stimulées par le fait de la distension mécanique du poumon.

Chez un animal trachéotomisé et portant dans la trachée une canule en T, Breuer distend fortement le poumon par

(1) Breuer. Die Selbstewerung der Athmung durch den Nervus vagus. Sitzungsber. der K. Akad. z. Wien, 1868, p. 909.

(2) Guttmann. Archiv f. Anatomie, 1875, pp. $300-525$, Taf. XV.

Rosenbach. Studien über den Nervus ragus. Berlin, 18i 7 .

Lockenberg. Verhandlungen der Würzb. phys.-med. Gesellschaft, 1873 (cité par Rosenbach). 


\section{(15)}

une ou plusieurs insufflations énergiques, il maintient le poumon distendu en fermant le tube par lequel il a pratiqué l'insufflation et qui se rend à l'une des branches de la canule en $\mathrm{T}$, l'autre branche est en rapport avec un manomètre élastique de Fick qui inscrit la courbe de la pression latérale sur le cylindre du kymographe. A la suite de la distension pulmonaire ainsi produite, Breuer observe une suspension des mouvements respiratoires qui peut durer pendant un temps assez long et qui est suivie ou accompagnée dès le début d'une expiration active extrèmement prolongée. Dans le premier cas le tracé de la pression trachéale reste horizontal pendant quelque temps, puis se relève peu à peu, dans le second il se relève dès le début. Après la section des pneumogastriques, la distension physique du poumon n'a plus d'effet sur le rhythme respiratoire.

Guttmann (1), opérant également avec le manomètre de Fick, a observé l'arrêt respiratoire survenant à la suite de l'insufflation pulmonaire, mais il nie l'existence de l'expiration active. Pour lui le tracé de la pression dans la trachée reste exactement horizontal; s'il se relève parfois un peu, cela devrait être attribué à la dilatation de l'air renfermé dans l'appareil, dilatation due à l'échauffement au contact des poumons et à une saturation plus complète de vapeur d'eau.

J'ai répété cette expérience de Breuer un grand nombre de fois en employant la même disposition expérimentale que dans l'expérience de Traube. Le tambour à levier de Marey y remplace avantageusement le manomètre de Fick

(1) P. Guttmann. Zur Lehre ron den Athembewegungen. Archiv für Anatomie, 1875. 


\section{( 16 )}

employé par Breuer et par Guttmann. Je me suis facilement convaincu de l'arrêt en expiration, de la suspension des mouvements d'inspiration qui survient quand, après une ou plusieurs insumpations énergiques, on ferme le tube d'arrivée de l'air de façon à maintenir les poumons distendus.

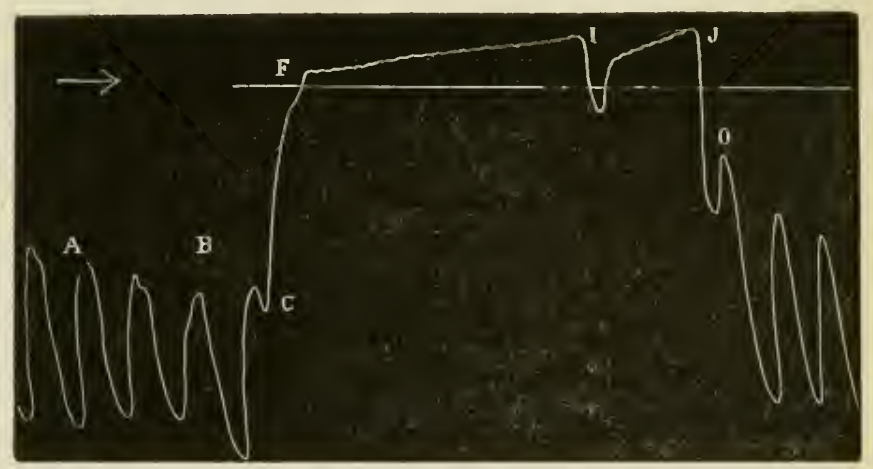

Fig. J. Inspiration coupe el expiration prolonge par le fait de la distension pu:monaire. De $\mathbf{A}$ en $\mathbf{B}$, respiration normale; en $\mathbf{G}$ une insuffation; en $\mathbf{F}$ on forme le tube; en $\mathbf{O}$ on ouvre de noureau le tube qui parl de la canule trachéale.

La figure כ̌ en montre un exemple. De A en B se roit un tracé normal de la respiration. La partie inférieure du trace correspond naturellement aux diminutions de pression dans la trachée, c'est-à-dire aux inspirations; la partie supérieure, aux expirations. En G, au moment où l'animal commence un mouvement d'inspiration, on fait une seule insufflation, la courbe de la pression latérale se relève naturellement jusqu'en $\mathrm{F}$, point où l'on maintient les poumons à l'état de distension en fermant le tube de la canule trachéale. Aussitôt la respiration s'arrête pendant plusieurs secondes, de F en I. De F en I, la courbe se relève, ce qui indique que l'état d'expiration dans lequel se trouve l'ani- 
mal est un état actif. Les muscles abdominaux se contractent en effet pendant cette pause respiratoire, comme on peut s'en convaincre par l'inspection directe : ces mouvements se communiquent à la peau et aux poils du ventre. Ceci est entièrement conforme à ce que Breuer et après lui Rosenbach ont décrit. En I l'animal fait une première, en $\mathrm{J}$ une seconde inspiration; en 0 on ouvre le tube trachéal et la respiration normale de l'animal reprend immédiatement.

J'ai répété cette expérience un très-grand nombre de fois sur au moins une douzaine de lapins et j'ai toujours obtenu des résultats très-concluants. Parfois cependant (chez les lapins chloralisés), j’ai obtenu comme Guttmann des arrêts respiratoires en expiration passive, c'est-à-dire que la courbe, au lieu de se relever, restait absolument horizontale jusqu'au moment où la première inspiration venait mettre fin à cette apnée par distension mécanique. Ce résultat, je l'ai surtout obtenu lorsque je maintenais le poumon modérément distendu, après l'avoir ventilé énergiquement par une série d'insufflations : l'apnée qui se produisait alors était une apnée mixte, due en partie à une oxygénation exagérée du sang, en partie à la distension physique du poumon. Dans les deux cas, que l'arrêt en expiration soit actif ou passif, les narines prennent pendant toute sa durée la position de l'expiration, elles restent fermées. Après la section des pneumogastriques, on n'obtient plus l'arrêt respiratoire par la distension pulmonaire.

Rosenbach, qui a observé également la contraction des muscles abdominaux pendant l'apnée par distension, considère cette contraction comme un phénomène tout à fait accessoire et local, dû à une action directe de la distension thoracique et abdominale sur les muscles de la paroi 
abdominale. Je crois que c'est là une erreur : pour moi la contraction des muscles abdominaux rentre bien ici dans le rhythme des mouvements respiratoires, elle fait partie de la phase d'expiration. En effet la suppression de la roic par laquelle le centre des mourements respiratoires commande à ces muscles, supprime leur contraction : j'ai pratiqué la section de la moelle épinière à la région dorsale et je n'ai plus observé leur contraction, quoique les parois abdominales se laissassent distendre comme auparavant à chaque insufflation.

Cette expérience de Brever el d'autres analogues semblent donc bien établir qu’à côté des fibres centripètes inspiratoires admises par la plupart des physiologistes, le pneumogastrique en contient également qui ont un effet opposé, qui suspendent l'inspiration et provoquent l'expiration (passive on active). Ces fibres proviendraient du poumon on de la plèvre et seraient excitées, entreraient en action dès que la distension mécanique du parenchyme pulmonaire atteint une certaine limite. Chez un animal à pneumogastriques intacts, toute inspiration doit donc fataleınent s'arrêter d'elle-mème à un niveau déterminé : ces fibres d'arrêt ne fonctionnant plus lorsque les pneumogastriques sont coupés, on comprend que les inspirations soient plus profondes, aillent pour ainsi dire jusqu'au hout chez les animaux qui ont subi cette opération.

Voyons ce qui arrive lorsqu'on essaye de mettre en évidence l'action de ces deux ordres de fibres les unes inspiratoires, les autres expiratoires dans le tronc du pneumogastrique. L'expérience a certainement été tentée des centaines de fois. Un très-grand nombre de physiologistes se sont occupés de l'influence qu'exerce l'excitation artificielle du bout central du pneumogastrique sur le centre des mouvements respiratoires. Malheurensement, les résul- 
tats auxquels ils arrivent sont extrêmement contradictoires. Je n'ai pas l'intention de refaire ici l'historique de cette question qu'on trouvera exposée tout au long dans les mémoires de Rosenthal et de Rosenbach.

Le plus grand nombre des expérimentateurs admettent avec Traube et Rosenthal que l'excitation du bout central faite dans des conditions convenables ne peut qu'exagérer les mouvements d'inspiration; augmenter leur nombre si l'excitation est faible, produire un véritable tétanos de l'inspiration si l'excitation est forte. Les expirations que l'on obtient parfois par l'excitation électrique du bout central du pneumogastrique doivent, d'après eux, être attribuées à des dérivations du courant électrique, allant atteindre, par exemple, le nerf laryngé supérieur dont l'excitation provoquerait l'expiration (1).

(1) Traube. Symplome der Krankheiten des Respirations u. Circulations apparates, p. 47.

Traube. Medicin. Zeitung des Vereins für Heilkunde in Preussen, 1847, n 5, p. 20.

Kölliker u. H. Müller. Würzb. Verh., 183ั4, V, p. 250 J.

Snellen. Onderzoekingen gedaan in het physiologisch Laboralorium der. Utrechtsche Hoogeschool. Jaar VII. Utrecht, 183̋4-185ั5̄, p. 121.

Claude Bernard. Leçons sur la physiologie el la pathologie du système nerveux, II, p. 582.

Funke. Lehrbuch, 3̄e Aufl., II, p. ड̃28.

Schiff. Lehrbuch, I, p. 412.

Gilschrisl. The british and foreign medico-chirurgical Reriew, 1858, XXII, p. 49ั.

Lindner. De nervorum vagorum in respirationem efficacitate. Diss inaug. Berol., 18 zั4.

Löwinsohn. Experimenta de nervi ragi in respirationem vi et effectu. Diss. inaug. Dorpali, 1838.

A. Waller et J.-L. Prévost. Etude.... de la déghutition. Archives de physiologie, 1870, pp. $183 \mathrm{~s}, 545$.

Rosenthal. Die Athembewegungen. Berlin, 1862. 
Cette opinion était assez généralement admise il n'y a pas longtemps : elle est encore enseignée dans la plupart des traités classiques de physiologie (1).

D'autres physiologistes ont obtenu tantôt une augmentation du nombre des mouvements respiratoires, tantôt un tétanos inspiratoire, tantôt, au contraire, un arrêt en expiration, suivant la force du courant employé et quelques autres circonstances accessoires. L'excitation de tout autre nerf sensible pourrait produire la même variété d'effets (2).

Enfin pour le plus petit nombre, le pneumogastrique ne contiendrait que des fibres expiratoires (5).

Dans une question aussi controversée, il ne me restait qu’à répéter les expériences un grand nombre de fois en

(1) Rosenthal s'est depuis convaincu que le nerf récurrent contient sonvent des fibres centripètes expiratoires (Bemerkungen, etc., über die Athembewegungen. Erlangen, 1875).

(2) $v$. Helmolt. Ueler die reflectorischen Beziehungen des Nervus vagus zu den motorischen Nerren der Athemmuskeln. Inauguraldiss. Giessen, 1856.

Aubert. Moleschoti's Untersuch. 1857. 11I, p. 272.

Tschishwitz. Nervis vagis irritatis diaphragma num in inspiratione an in exspiratione sistitur? Diss. inaugur. Vratisla viae, 1857.

Burliart. Pflüger's Archiv. Bd. I, p. 10i.

Paul Berl. Des effets de l'excitation du nerf pneumogastrique, du nerf larynguẻ supérieur et du nerf nasal sur la respiration. Archives de physiologie, 1869, pp. 179-522.

Burkart. Studien über die automalische Thätigkeit des AthemernIrums. Pflüger's Archiv, p. 427, XVI.

(3) Budge. Comples rendus, 1854, XXXIX, p. 749.

Ousjanikow. Virchow's Archiv., 1860, XVIII, p. 572 .2.

Eckard.

Rosenbach. Studien über den Nervus ragus.

Rosenbach, daus une Note publiée ultérieurement dans les Archives de Pnüger, p. 502, XVI, a reconnu qu'il arait été induit en erreur, et s'est rallié aux idées de Rosenthal et de Traube sur ce point spécial. 


\section{( 21$)$}

m'entourant de toutes les précautions, et surtont sans parti pris, c'est-à-dire en cherchant à me désintéresser autant que possible du résultat que j'allais obtenir.

Voici comment je dispose l'expérience.

Pour exciter électriquement le pneumogastrique j'emploie une pile Grenet et le chariot de Du Bois-Reymond. Dans le circuit primaire (celui qui va de la pile au chariot, j'intercale une clef de Du Bois et un signal électrique Deprèz qui par ses vibrations inscrit sur le cylindre enregistreur la durée du passage du courant électrique. Le nerf repose sur deux lames de platine supportées par une plaque de verre et reliées à la bobine secondaire (induite) du chariot.

Les électrodes et la plaque ne touchent pas l'animal : le bout coupé du nerf offre donc entre la plaque et le corps de l'animal une portion suspendue en l'air, formant pont. Dès qu'on actionne la clef, le courant passe et le signal électrique inscrit ses vibrations tant que dure l'excitation du nerf. J'enregistre à côté les mouvements respiratoires. La méthode la plus parfaite de représenter ces mouvements daus le cas qui nous occupe, c'est, je crois, d'indiquer leur effet utile, c'est-à-dire les quantités relatives d'air qui entrent et qui sortent à chaque mouvement.

Si l'animal respire par une canule en rapport avec une atmosphère confinée, il suffira d'inscrire les variations de pression que subit cette atmosphère pour pouvoir en déduire les volumes relatifs d'air qui entrent et qui sortent à chaque excursion de la poitrine de l'animal. L'air que l'aninal respire est renfermé dans une grande bouteille (4 à 12 litres de capacité) bien bouchée à l'aide d'un bouchon de caouctchouc. Deux tubes de verre coudés traversent le bouchon, l'un est relié par un caoutchouc très- 
court à la canule trachéale de l'animal, l'antre transmet à distance par un tube de caoutchouc les variations de pression de la bouteille et par conséquent la respiration de l'animal, à un tambour enregistreur de llarey. Le style écrivant lu tambour el celui dı signal Deprèz tracent leur courbe en regard l'une de l'autre.

Tout mouvement d'inspiration va raréfier l'air de l'appareil, déprimer la membrane du tambour enregistreur, rapprocher la plume écrivante de l'abscisse, tout mouvement d'expiration aura un effet inverse, gonflera. la memlrane du tambour et élèvera la plume (1).

Les choses étant ainsi disposées, les pneumogastriques étant coupés, le bout central de l'un d'eux reposant sur les électrodes, fermons la elef; cu employant un courant d'intensité moyenne, nous obtiendrons généralement l'effet indiqué par Rosenthal, c'est-à-lire un effet inspiratiore: tétanos inspiratoire ou série de contractions du dia. phragme dans lintervalle desiguelles celte cloison musculaire ne se relàche pas compićtement. On peut observer tous ces intermédiaires entre ces tétanos el une simple accélération de la respiration. La question a été trop bien éludiéc par d'autre pour que je m’y arrête ici longuement. Je me borne à donner un tracé de ce genre comme terme de comparaison avec ceux qui vont suive. Dans la fig. 6 , on trouve de $\mathrm{A}$ en $\mathrm{B}$ un tracé normal de la respiration; de $\mathrm{B}$ cn $\mathrm{C}$ excitation d'un pneumogastrique par un courant d'intensité moyenne produisant une série de petites inspi-

(1) Ce mode d'inscription des mouvements respiratoites est indique par Beaunis (Traité de plsysiologie), comme ayant été imaginé par Bert. Il faut renouveler l’air de la bouteille pour peu que l'expérience se prolonge. 


\section{( 25 )}

rations se succédant si rapidement que le thorax ne peut prendre la position d'expiration dans l'intervalle. De C en $D$ on cesse l'excitation, l'animal reprend sa respiration, mais l'excitation du pneumogastrique a laissé une légère tendance à la prédominance du type inspiratoire.

D’antrefois j'oltiens un effet tout opposé, un arrêt respiratoire en expiration. Chez certains animaux, en faisant varier la force du courant, je produis tantôt un arrêt en expiration, tantôt un tétanos inspiratoire, et cela que les sujets soient anesthésiés ou non. Ces expériences ont été faites sur des lapins, des cobayes, un chien et un chat. Chez le chat l'excitation du pneumogastrique a toujours provoqué un arrêt en expiration, jamais d'inspiration.

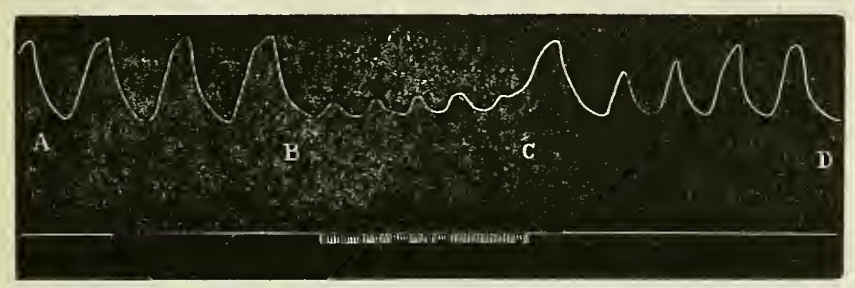

Fiy. 6. Effet ordinaire de l'excitation électrique du pneumogastrique (boul central) Prédominance du type inspiratoire.

Au lieu de l'excitation électrique, j’ai fréquemment employé des excitants de nature physique ou chimique: sections brusques, répétées, froissement du nerf entre les mors d'une pince, contact avec une solution saturée de $\mathrm{NaCl}$, elc. Ces moyens produisent des effets du même ordre que l'électricité.

Nous sommes donc amenés de nouveau à cette conclusion que si la majeurc partie des fibres centripètes respiratoires du pneumogastrique se rendent à un centre 


\section{( 24$)$}

d'inspiration, il en est d'autres dont l'excitation produit un effet tout opposé, agit sur un centre d'expiration.

Il est impossible de séparer anatomiquement ces deux ordres de fibres. Les effets de, cette dissection que le scalpel est impuissant à réaliser, on peut les obtenir au moyen de substances toxiques dont l'action se localise sur l'un de ces faisceaux de fibres. J'ai rencontré une substance, l'hydrate de chloral, qui précisément a pour effet (chez le lapin) de diminuer l'action des fibres inspiratrices du pneumogastrique, ou plutôt sans doute de déprimer l'excitabilité du centre auquel abontissent ces fibres (1).

Le chloral supprimant l'action des fibres inspiratoires, celle des fibres expiratoires devient prédominante. Cliez un animal empoisonné par le chloral, on n'observe plus cette diversité d'effets à la suite de l'excitation du bout central du pneumogastrique coupé. Toute excitation du nerf faite dans ces conditions a pour effet de suspendre les mouvements respiratoires, de produire un arrêt en expiration. Il faut pour cela une action profonde du chloral, l'animal doit être non anesthésié, mais réellement empoisonné (2). Une injection de 2 à 3 grammes de chloral

(1) On sait depuis plusieurs annces que le chloral a pour effet de faire baisser le nombre des mourements respiratoires (Lichreich, Rajewsky, 1/c Rae, Rokitansky, v. Mering).

(2) Burkart (Pflüger's Archiv XV1, p. 481) attribue au chloral une action toute différente. Les animaux chloralisés n'offraient plus dans ses expériences que des fibres inspiratrices dans le pneumogastrique.

"Sobald durch Morphium oder Chloralhydrat eine tiefe Hirn-Narkose " bei dem Versuchsthier eingeleitet ist, hedingt die Reizung des centralen - Vagusstumpfes unterhalb des Abganges des Nervus laryngeus sup. nur „ melir inspiratorische Erscheinungen. " Il en conclut que les fibres exp iratrices contenues dans le tronc du pneumogastrique cervical n'agissent sur' le nœud vital que par l'intermédiaire des hémisphères cérébraux. La 
(dissous dans trois ou quatre fois son poids d'eau) dans le péritoine chez un lapin de taille moyenne permet en général d'arriver à ce stade où la respiration se ralentit extraordinairemeni, et où la mort est imminente. C'est dans les quelques minutes qui précèdent le dernier mouvement respiratoire de l'animal qu'on obtient des résultats absolument constants. Toute excitation mécanique, chimique ou électrique arrête la respiration en expiration; celle-ci reprend dès que l'on suspend l'application de l'excitant.

La fig. 7 en montre un exemple que l'on fera bien de comparer avec le résultat indiqué dans la fig. 6. De A en B respiration normale très-ralentie, de $B$ en $C$ excitation électrique d'un pneumogastrique, arrêt en expiration ; en $\mathrm{C}$ on cesse l'excitation, la respiration reprend immédiatement; en D nouvel arrêt respiratoire par excitation du pneumogastrique. On remarquera que la première inspiration qui suit chaque arrêt respiratoire a une. durée plus forte que les autres.

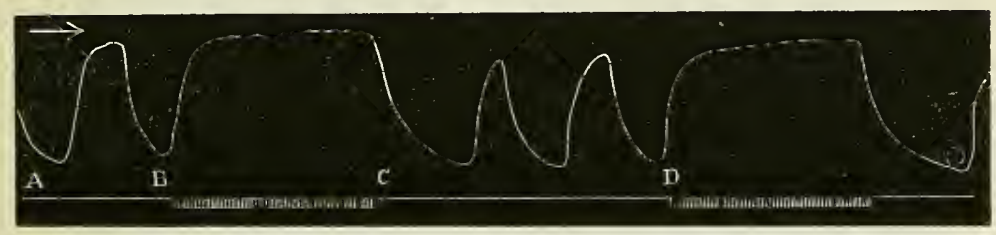

Fig. 7. Lapiu empoisonné par le chloral. Effets de l'excitation d'un pneumogastrique. Arrét en expiralion.

conlradiction qui exisle entre les expériences de Burkarl el les miennes s'explique en parlie au moins par les conditions différentes dans lesquelles elles onl été instituées. Burkart, tenant à conserver l'animal en vie pendant l'expérience, ne lui injectait qu'une faible dose de chloral.

Dans mes expériences, il s'agit toujours d'un empoisonnement par le chloral se terminant par la mort. Les résultals de mes expériences sont en contradiction flagrante avec la conclusion que Burkart tire des siennes. 


\section{(26)}

Les résultats obtenus de cette façon présentent un tel degré de constance, que l'on peut, en ouvrant et en fermant la clef intercalée dans le circuit électrique, modifier à son gré le rhythme respiratoire de l'animal. La fig. 8 est empruntée à une expérience ou pendant une minute entière on a produit alternativement une expiration longue, une brève, une longue, etc., à l'aide de cette manœuvre de la clef électrique.

Si le début de l'excitation correspond à la plıase expiratoire de l'animal, celle-ci se prolonge pendant toute la durée de l'excitation. On peut ainsi suspendre la respiration pendant plus d'une minute. $\mathrm{Si}$ l'on prolonge trop longtemps l'excitation, l'animal ne se remet plus à res-

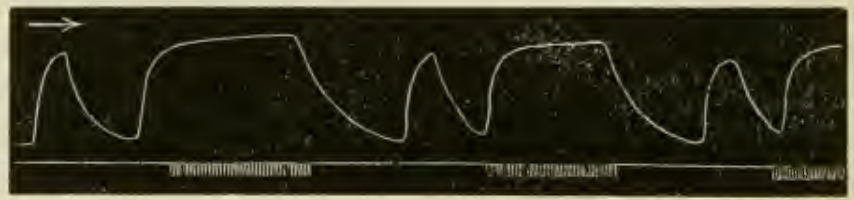

Fig. \& Lapin empoisomuc par le chloral. Klsythme respiratuire modifie par des excilations fréquenles du pneumogastrique.

pirer, on paralyse complétement son centre inspiratoire, il est mort. La ligne horizontale de l'expiration se continue alors avec celle de la mort, ce qui prouve que dans ce cas au moins il s'agit d'une expiration passive, c'est-à-dire

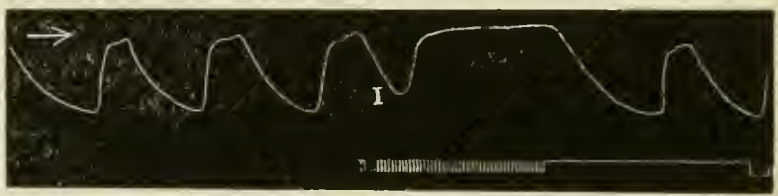

Fig. 9. Lapin chloralisé. Effets de l'excitation èlectrique d'un pmeumogastrique survenant au moment d'une inspiration I. 
d'une simple action d'arrêt sur le centre inspiratoire (voir la planchie I, dernier tracé). Si le début de l'excitation correspond à l'inspiration, l'animal ne s'arrête pas en route, il complète cette inspiration, mais en l'abrégeant comme le montre la fig. 9.

Les petites ondulations (1) qui se voient sur la courbe de l'arrêt respiratoire correspondent aux pulsations cardiaques. Tous ces faits et d'autres peut-être qui m'échappent se voient beancoup mieux encore sur les graphiques de la planche I.

Le pneumogastrique semble donc contenir deux ordres de fibres respiratoires centripètes : des fibres inspiratoires et expiratoires. Ces fibres nerveuses sont probablement des conducteurs indifférents, ne différant entre elles que par leur point d'arrivée, parce qu'elles aboutissent à des groupes distincts de cellules nerveuses de la moelle a!longée; et le chloral aggit sans doute non sur des fibres inspiratrices, mais seulement sur les cellules ncrveuses auxquelles ces fibres se rendent. Nous sommes ainsi amenés à considérer dans la moelle allongée un centre inspiratoire et un centre expiratoire, le chloral agissant pour paralyser le premier. Le chloral à haute dose a pour effel de ralentir extrêmement les mouvements respiratoires qui peu à peu cessent complétement, bien avant que le cœur ait suspendu ses battements. Le tracé suivant en est un exemple. Il représente la pression dans la trachée d'un lapin empoisonné par le chloral, environ une minute

(1) Le graveur a négligé de reproduire ces ondulations dans la plupart des graphiques. Elles ont élé indiquées, mais peu exactement, dans la figure 5. 


\section{( 28 )}

après le dernier mouvement respiratoire. Les petites ondulations correspondent aux pulsations du cœur.

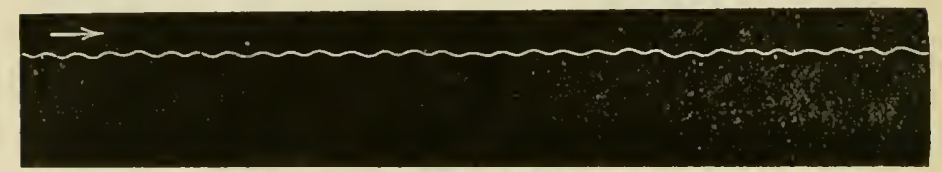

Fig. 10. Lapin empoisonne par le chloral. Pression dans la trachè. Persistance des pulsations cardiaques alors que la respiration a cesse.

Dans les circonstances ordinaires, l'expiration est absolument passive, due uniquement à l'élasticité du thorax et des viscères abdominaux; l'expiration n'est en général que la suspension de l'inspiration. Aussi le centre expiratoire a d'ordinaire un rôle absolument passif vis-à-vis du système nerveux périphérique centrifuge. Son action normale paraît donc être une action d'arrêt à l'égard du centre inspiratoire (1).

Ce n'est que dans des circonstances spéciales (vénosité exagérée du sang, dyspnée) que ce centre élargit son cercle d'action, et met en jeu les fibres nerreuses motrices qui vont aux muscles expirateurs. Lexpiration devient alors active.

J'ajouterai que j’ai essayé d'exciter les fibres respiratoires centripètes du pneumogastrique, par l'introduction de substances irritantes (alcool) dans la cavité pleurale. Je. n'ai pas obtenu de résultats dignes d'être notés.

(1) Voir : Rosenbach, loc. cit., p. 76. 


\section{EXPLICATION DE LA PLANCHE.}

Graphique de la respiration chez un lapin empoisonné par le chlora pendant les sept minutes qui ont précédé la mort. L'animal respire dans une atmosphère confinée dont on inscrit les variations de pression. Les [neumogastriques ont été coupés, on excite le boul central du pneumogastrique droil par une série de chocs d'induction (chariot de Du Bois) d'intensité moyenne, dont le signal électrique donne un graphique (tracé inférieur de chaque ligne). Arrêts respiratoires en expiration. A la suite du dernier arrêt respiratoire, l'animal fait une seule inspiration superficielle, puis cesse de respirer et meurt. (Ligne VII, D.)

Le tracé se lit de gauche à droite et de bas en haut. Chaque ligne représente une durée d'une minute environ, elles se suivent arec quelques secondes d'interruption seulement.

La distance qui sépare les deux styles écrivants ayant varié un peu pendant l'expérience, le graphique du signal électrique ne saurait servir d'abscisse que pour chaque ligne prise isolément.

I, Il, III..... $1^{\mathrm{re}}, 2^{\mathrm{me}}, \mathrm{J}^{\mathrm{me}} \ldots . .$. minute.

Tracé I. Ins. Inspiration.

Ex. Expiration.

de A en B Excitation du pneumogastrique droit.

A. Ex. Arrêt en expiration.

I. P. Inspiration prolongée.

$A^{\prime} B^{\prime}$. Excitation du pneumng. en $A^{\prime}$ inspiration coupée.

Tracé 11I. I. C. Inspiration coupée.

Tracé VII. en C. Excitation du pneumog. jusqu'en

D. Dernière inspiration, puis l'animal cesse de respirer.

On remarque de 1 en VII une diminution graduelle de l'amplitude des mouvements respiratoires (à mesure que l'empoisonnement fait des progrès). 

L'IN

\section{Minthin}

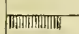

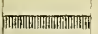




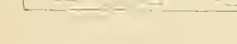

$$
\text { c }
$$
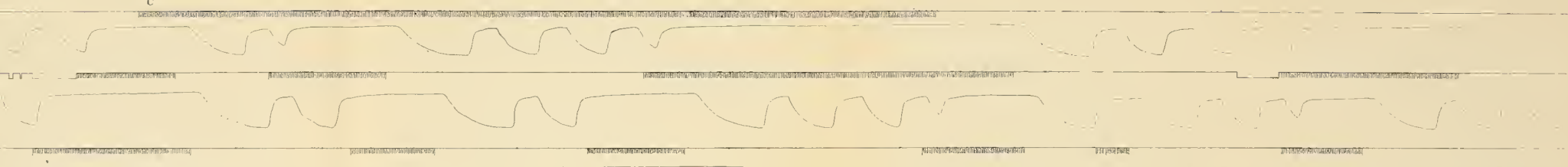

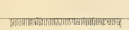

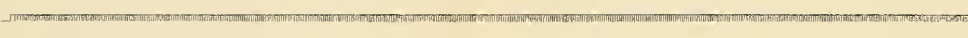

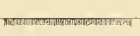

"मw

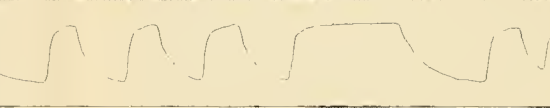

-
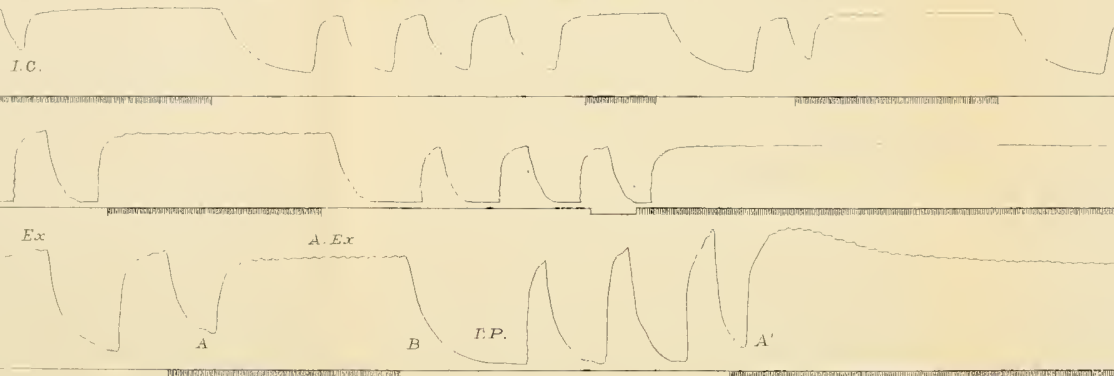

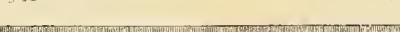







\section{COLUMBIA UNIVERSITY}

This book is due on the date indicated below, or at the expirntion of a definite period after the date of borrowing, as provided by the rules of the Library or by special arrangement with the Librarian in charge.

\begin{tabular}{|c|c|c|c|}
\hline DATE BORROWED & DATE DUE & DATE BORROWED & DATE DUE \\
\hline & & & \\
\hline & & & \\
\hline & & & \\
\hline & & & \\
\hline & & & . \\
\hline & & & \\
\hline & & & \\
\hline & & & \\
\hline & & & \\
\hline & & & \\
\hline & & & \\
\hline & & & \\
\hline & & & \\
\hline & & & \\
\hline & & & \\
\hline & & & \\
\hline C28 638/M5O & & & \\
\hline
\end{tabular}




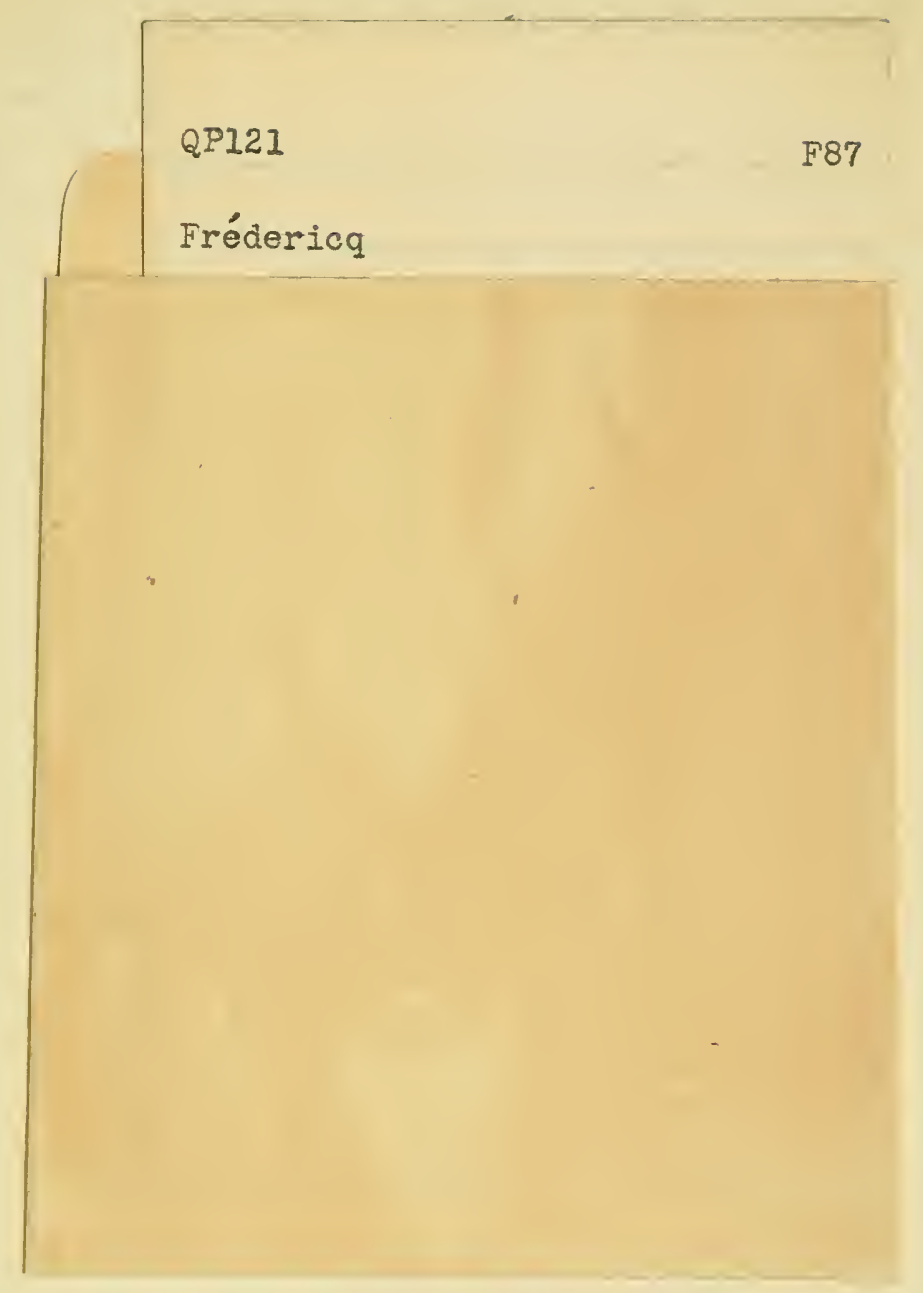


